\title{
Surgical Perspectives for Pediatric Theater Teams during the SARS-CoV-2 Pandemic and Beyond: Narrative Review and Mandatory Tasks Guidelines
}

\author{
${ }^{1}$ Department of Paediatric Surgery, Chelsea and Westminster \\ Hospital NHS Foundation Trust, London, United Kingdom \\ 2 Department of Pediatric Anesthesia, Chelsea and Westminster \\ Hospital NHS Foundation Trust, London, United Kingdom \\ ${ }^{3}$ Department of Pediatric Surgery, Chelsea Children's Hospital, \\ Chelsea and Westminster Hospital NHS Fdn Trust, Imperial College \\ London, London, United Kingdom
}

Roberta lacona ${ }^{1}$ Kiran Chima ${ }^{2}$ Ami Kotecha ${ }^{2}$ Amulya Saxena ${ }^{3}$

Eur J Pediatr Surg 2021;31:305-310.

\begin{abstract}
Address for correspondence Roberta lacona, MD, Department of Paediatric Surgery, Chelsea and Westminster Hospital NHS Foundation Trust, Hills Road, London SW10 9NH, United Kingdom (e-mail: roberta.iacona@doctors.org.uk).
\end{abstract}

\begin{abstract}
Keywords

- COVID-19

- pediatric

- theater teams

- surgery

- anesthesia

- protection

This is a narrative review during the ongoing coronavirus disease 2019 (COVID-19) pandemic to streamline workflow of pediatric surgical patients in operating theaters and for theater teams involved in their management. Pediatric patient anxiety in theaters, aspects of communication, and optimizing vision during surgery during the pandemic have also been addressed. The COVID-19 pandemic has led to the creation of pathways in the surgical management of patients. As the pandemic progressed, hospitals developed pathways to offer increased protection to staff during procedures. This narrative review provides a clear perspective in the management of pediatric patients in operating theaters. Guidelines received from National Health Authorities and Societies affiliated with surgery, endoscopic surgery, anesthesiology, and endoscopy were carefully reviewed regarding their recommendations and data emerging from reports on COVID-19 were selected to compile the pathways specific for pediatric patients and staff. The workflow pathways have been successfully implemented during the pandemic and include a section on patients for endoscopy as well as approach to endoscopic surgery and open procedures. Theater room ergonomics that were successful during the pandemic have been outlined along with identification of areas specific to the pediatric patient anxiety, interteam communication/identification, and visor-related vision. The guidelines used successfully during the pandemic for pediatric theater teams can be used or adapted for formulating local hospital guidelines in other centers that could be valuable in patient management beyond the pandemic.
\end{abstract}

\section{Introduction}

Over the past year, the spread of the novel coronavirus infection by severe acute respiratory coronavirus 2 (SARSCoV-2) has affected the global population. On March 11, 2020, the World Health Organization (WHO) officially declared coronavirus disease 2019 (COVID-19) a pandemic, thereby drastically changing our way of approaching patients in the shadow of this disease. While the vast majority of infected adults will present with respiratory symptoms such as fever, received

April 23, 2021

accepted

May 10, 2021

published online

June 20, 2021 (c) 2021. Thieme. All rights reserved.

Georg Thieme Verlag KG,

Rüdigerstraße 14,

70469 Stuttgart, Germany
DOI https://doi.org/

10.1055/s-0041-1731293.

ISSN 0939-7248. 
cough, myalgia, or fatigue, ${ }^{1-3}$ a recent meta-analysis showed that $17.6 \%$ of COVID-19 positive pediatric patients will present with milder symptoms, including nausea, vomiting, diarrhea and abdominal pain, leading to challenges when it comes to their approach, management, and risk stratification. ${ }^{4}$

The American College of Surgeons published the "COVID19 Guidelines for Triage of Pediatric Patients" with the aim of optimizing hospital resources and preserving the health of providers. $^{5}$

The guideline has divided the most common pathologies into three categories:

(a) Emergency cases (life threatening-as per Royal College of Surgeons [RCS] stratification $)^{6}$

(b) Urgent cases (completed in days)

(c) Electives cases (minimal risk if delayed)

As most centers are in the midst of the pandemic, emergency procedures in pediatric and neonatal surgery are currently being performed using current Public Health England (PHE) advice regarding personal protective equipment (PPE). ${ }^{7}$ This has helped to risk stratify each patient encounter and guide the use of this precious resource. The categories describe appropriate PPE for reducing risk of infection from contact, droplet, and airborne transmission.

Clinical manifestations of SARS-CoV-2 in the pediatric age group have recently been reported to be less severe than adults, but children of all ages are susceptible, with infants in particular being more vulnerable to infections. ${ }^{8}$ The fecaloral transmission of SARS-CoV-2 suggests that asymptomatic children and adults may be shedding infectious virus (although the presence of viral particles may not directly result in infection as few researchers commented on the fact that they could not grow virus from the fecal samples suggested that these were digested viral fragments). ${ }^{9}$ of even greater concern is the fact that children have persistently tested positive on rectal swabs even after nasopharyngeal testing was negative. ${ }^{10}$ This is important for the management of children throughout the hospital, including the emergency department (ED), radiology department, operating theaters, and wards. During the early periods of the pandemic, there was a lag in receiving the results of the swab tests, and all pediatric patients for emergency surgery were considered SARS-CoV-2 positive until test results are received. However, the availability of newer and more reliable SARS-CoV-2 tests with rapid turnaround ( $\sim 1$ hour) has enormously assisted in stratified pathways of care. With the pandemic continuing in the near future, precautions including PPE for aerosol-generating procedures (AGPs) that are being undertaken during the peak of the pandemic will need to continue.

The ideal operating room conditions have been recently described to manage COVID-19 patients ${ }^{11}$; however, these conditions cannot be achieved worldwide and will have to be adapted in the global context. This is further complicated by the fact that in pediatric surgery extremely sick premature neonates require surgery on the neonatal intensive care unit (NICU) that is used as a makeshift operating room.

This narrative review outlines the approach for pediatric surgical patients at Chelsea and Westminster Hospital and clearly outlines (A) approach to pediatric surgical emergency procedures, (B) ergonomics of anesthesia, (C) step-by-step pathways of donning and doffing for the entire operating room staff in (i) single room operating theaters and (ii) NICU and (D) staff-communication and individual team challenges. Even when the pandemic comes to an end, the timing of which is an intense issue of debate, the procedural pathways will stay in place to protect the staff and to keep the transmission in check.

The current approach during the pandemic is to manage every pediatric patient as suspected COVID-19 positive from time of presentation to a healthcare setting. A nasal swab is performed as part of the admission process and the child is admitted to a dedicated suspected COVID-19 ward. When the child is ready for theater, only the primary caregiver is allowed to accompany the child and is escorted by a nurse in droplet precaution PPE (this includes: a surgical mask, plastic apron, gloves, hat and optional eye protection/ face shield). During transfer from the ward to the theater, the caregiver and child (if tolerated) wear a face mask. The caregiver also wears a plastic apron/ gown during transfer and, prior to entering theater, dons a theater hat and shoe covers.

\section{Approach to Pediatric Surgical Emergency Procedures}

\section{Endoscopy (Nonsurgical) Procedures}

The knowledge acquired so far on the contagion of the virus and how this is spread has pointed out how the aerosolizing procedures are the most dangerous. It is also alarming that $70.3 \%$ of patients who had stool viral RNA positive were negative on respiratory specimens. ${ }^{4}$ Because of the short distance between patients and operator and because of the prolonged contact with patient's secretions, endoscopic procedures represent a risk and need to be considered carefully. Recommendations to perform endoscopic procedures at the time of COVID-19, regardless of patient risk stratification, should involve a negative pressure room with the entire staff using contact and droplet precautions. Current guidelines of North American Society for Pediatric Gastroenterology, Hepatology \& Nutrition recognize three categories of risk ${ }^{12}$ :

(1) Emergency procedures: Indications include esophageal/gastric foreign bodies ingestion such as button batteries, magnets, sharp or toxic foreign bodies, management of massive gastrointestinal bleeding, endoscopic vacuum therapy for perforations/leaks, acute cholangitis, jaundice, secondary to malignant or benign biliary obstruction, acute biliary pancreatitis and/or cholangitis with stone and jaundice, infected pancreatic collections, urgent inpatient nutrition support-percutaneous endoscopic gastrostomy/nasojejunal tube, gastrointestinal obstruction needing urgent decompression/stenting.

(2) Urgent procedures: Procedures in which the benefits overweigh the risks (can be deferred for days or weeks).

(3) Elective procedures (defer until resumption of routine practice): These include the rest of the procedures. Beyond 
the peak of the pandemic when routine procedures are gradually phased in, more widespread access to testing may allow scaling back the level of PPE required.

While negative pressure ventilation is recommended to reduce the aerosol transmission of airborne agents, ${ }^{13}$ the vast majority of hospitals in the United Kingdom have positive pressure systems in their operating theaters, enabling regular air changes of between 20 and 25 per hour. PHE $^{14}$ advice until April 2020 suggested that $63 \%$ of circulating aerosol was cleared from an area of positive pressure ventilation with every air change and $>99 \%$ after 20 minutes. This 20-minute period is therefore thought to be a prudent compromise between reducing cumulative viral exposure for staff and working in positive pressure rooms. Since then, the time a room should be left empty after an AGP has been referred to as the "Aerosol Clearance Time" (ACT) to more accurately reflect the time required for aerosol clearance that is not only a function of the time but also the air exchange rate, $^{15}$

\section{Endoscopic Surgery Procedures}

Society of American Gastrointestinal Surgeons (SAGES) and the RCS have highlighted concerns that SARS-CoV-2 virions transmission could occur during endoscopic surgery posing a risk to operating theater staff. ${ }^{16-19}$ The concerns were mainly raised as hepatitis B virus particles in a previous study were identified in surgical smoke, ${ }^{20}$ although the scientific evidence regarding SARS-CoV-2 transmission is not evidence based. $^{21}$ There is conflicting guidance in electrosurgery, the use of which to date has not shown any evidence for an increase in disease transmission over the pandemic. ${ }^{22}$

SAGES has advised that laparoscopy is considered to carry some risk of aerosol-type formation and transmission of infection; however, the level of risk has not been defined. ${ }^{23}$ Caution has been urged more with the SARS-CoV-2 virus as it can remain viable and infectious in aerosols for hours and on surfaces up to days. ${ }^{24}$ Although it has been recommended if the risks outweigh the benefits, endoscopic procedures can be performed with the application of smoke filters to filter the pneumoperitoneum after the procedure; however, recent reports could not identify smoke filters and smoke evacuators specifically tested for SARS-CoV-2 virus. ${ }^{25,26}$ Besides this, leaks from port sites can occur during endoscopic surgery and if endoscopic surgery is considered as an urgent procedure, it would be also worth considering gasless options. ${ }^{27}$ Risk stratification due to availability of rapid detection kits has enabled restoration of endoscopic surgery

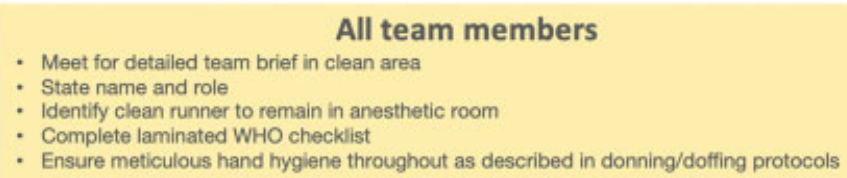

Anesthetic team

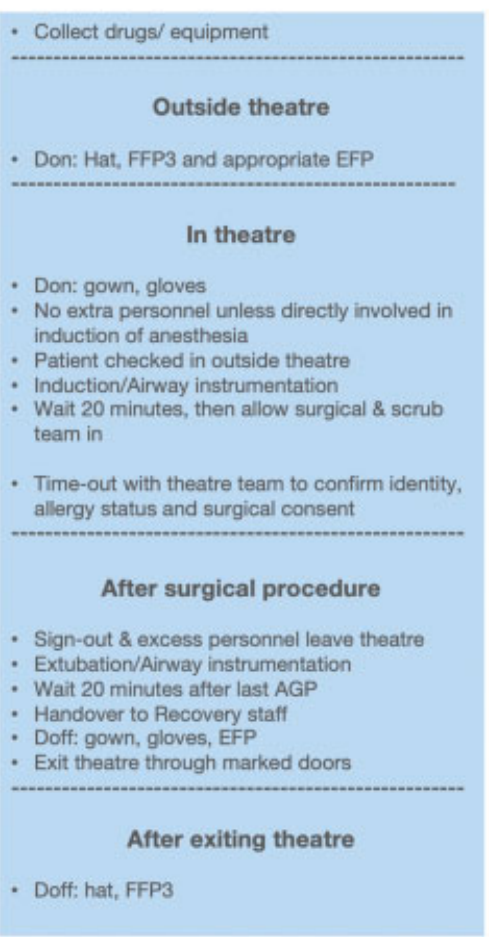

Surgical team

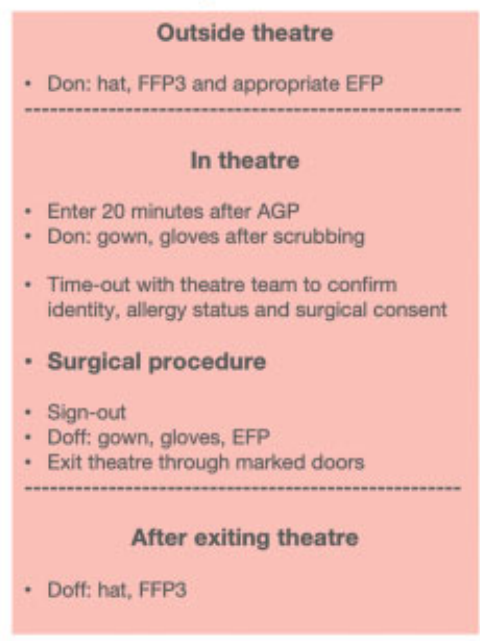

Scrub team

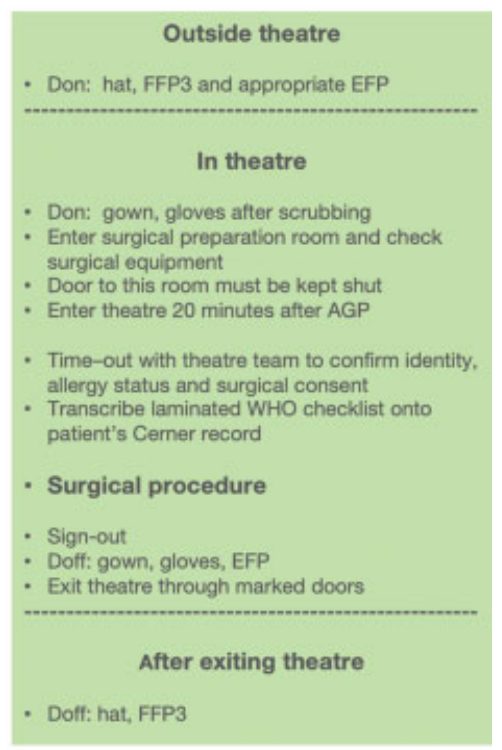

Fig. 1 Flow Chart: Theatre team Huddle and Time Out in COVID era. AGP, aerosol generating procedure; EFP, eye/face protection; FFF3, face filter piece. 
at our center in low-risk cohorts for emergency and urgent procedures. Elective endoscopic surgery procedures are still not being performed as there is a large-scale reallocation of staff away from theaters toward management of COVID-19 patients.

\section{Open Surgery Procedures}

All open surgical procedures are performed using airborne precaution level PPE keeping the operating time as short as possible to complete the procedures safely.

\section{Theater Room Ergonomics for Suspected or Confirmed SARS-CoV-2 Positive Child}

Guidelines have been devised and implemented at Chelsea and Westminster Hospital to maintain high standards of patient safety and minimize cumulative viral exposure for theater staff ( - Fig. 1). Many elements of the normal practice have been incorporated in this guidance to make it easier for staff to follow.

In our experience, thorough preparation is key. A dedicated "suspected/ confirmed COVID-19 + ve" operating theater has been assigned. This theater is also easy to isolate from the other theaters and routes commonly used by staff or patients from non-COVID-19 wards. The chosen theater was also closest to ED and easily accessed from the wards. It is vital to meticulously check that all the necessary surgical and anesthetic equipment required are present, as much of the stock had been moved at the peak of the pandemic to support critical care. All team members must be aware of the new pathways in use for this group of children. This pathway is described below.

\section{Team Brief}

1. Only the patient expected is discussed to avoid any confusion (patients are discussed in the anesthetic rooms that are used for the brief. No patients will access this room; therefore, it can be used as a "clean" area).

2. All team members state their names and roles clearly. The Surgical and Anesthetic Consultants must ensure that their trainees understand and accept their roles. For this group of children, it is advisable that the most senior and experienced clinicians lead the procedure to minimize the time taken to complete the procedure.

3. Minimize the number of people in theater to reduce the viral exposure for each individual staff member.

4. A "clean runner" must be identified and remain in the anesthetic room in case any further equipment or help is required. This will most likely be a member of the nursing team, so named personnel to call when help is required should be agreed and discussed in advance.

5. Discuss level of PPE required for each member of the theater team to ensure staff safety and reduce wastage.

6. Clear communication proved a challenge in PPE early on in the pandemic and so the timing of the WHO Surgical Safety Checklist (SSC) was adapted. The checklist is now undertaken at the brief using a printed and laminated checklist that is taken into theater once completed. Once the child arrives in the operating theater and their identity, allergy status, and surgical consent are confirmed, the information on the checklist is transcribed to their electronic notes by a member of the theater team. The laminated sheet itself is wiped clean at the end of the case using an alcohol-based wipe, once inside the operating theater and a second time when it is taken outside. It can then be used again.

\section{Entry into Operating Theater}

1. Access for the child and carer is directly into the operating theater through a set of doors that opens into theaters from the main corridor. Both child and carer wear surgical masks during transfer from the ward. The carer also wears a plastic gown and gloves during transfer.

2. Before entering the operating theater, the carer is asked to put on a hat and shoe covers. As the child is usually transferred on a trolley, any porters or nursing staff accompanying the child also wear droplet precaution PPE.

3. Access for theater staff is through a single door that leads into the operating theater past the washbasins used for scrubbing.

4. Before entry, all staff don a hat, fit-tested filtering facepiece class 3 (FFP3; or reusable) mask, and appropriate eye protection. There is a donning station at the door with all the above PPE.

5. Immediately on entering the scrub area, staff perform hand hygiene and then don a long-sleeved gown (or alternative coverall) and gloves.

6. The nursing scrub team enters first and scrub and complete the donning process. They then enter the surgical preparation area that has a door. This door will remain shut while they check their equipment.

7. The anesthetic team enters next, complete the donning process, and check their equipment. Equipment and medication required for the procedure are brought in on trollies or trays that can be wiped clean at the end of the case.

8. The surgical team only enters once the child is anesthetized and 20 minutes have passed after any AGPs have been completed.

9. The clean runner will wait in the anesthetic room in case any further help or equipment is required.

\section{Process in Theater}

1. The scrub team remains in the surgical preparation area with the door shut until 20 minutes after any AGP.

2. The child enters the operating theater directly from the theater corridor along with the carer. The child's identity, allergy status, and consent for surgery are confirmed at the door and the nurse remains outside theater.

3. The carer is allowed to remain during the induction of anesthesia if they wish.

4. Once the child is anesthetized, the carer is asked to leave through the same set of doors that they entered from. They are escorted back to the ward by the ward nurse.

5. Only the anesthetic team (consultant, trainee and operating department practitioner) is present for the induction 
of anesthesia. A timer is started to accurately record 20minute periods after the last AGP and then allow the scrub team and surgical team to enter.

6. The theater team again confirms the child's identity, allergy status, and consent for surgery before proceeding. The WHO SSC can now be transcribed to the patient's electronic note record.

\section{Process in Theater on Completion of Procedure}

1. Once the procedure is complete, the surgical and scrub team follow the doffing process and leave theater. Only the team members who are required to directly assist in airway management during extubation remain.

2. Particular attention is paid to choosing a technique for extubation that reduces the risk of coughing. Suctioning should be performed prior to extubation.

3. Recovery staff enter in droplet precaution PPE 20 minutes after the last AGP. The child is recovered in theater to reduce the risk of contamination of other clinical areas.

4. The anesthetic staff doff and leave theater once they have handed over the care of the child to the recovery staff.

5. The clean runner must remain in the anesthetic room until the child leaves theater.

6. The child's parent/carer is allowed to stay with the child during the recovery period and accompany the child back to the ward.

\section{Surgical Team Pathway}

\section{Single-Room Operating Theaters}

1. Preprocedure: Before entry, all surgical staff must don a hat, fit-tested FFP3 (or reusable) mask, plastic apron, appropriate eye protection, and gloves after hand hygiene. There is a donning station at the door with all the above PPEs using a 360-degree buddy check system to ensure proper donning.

2. The surgical team will again confirm the child's identity, allergy status, and consent for surgery before proceeding.

3. Following this the team proceeds to the scrub area and removes the plastic apron and gloves. The staff then scrubs and dons a long-sleeved gown and sterile double gloves.

4. Postprocedure: Once the procedure is completed, the surgical team will perform doffing in the theater room close to the exit (removal of gown and one pair of gloves, while retaining the FFP3 mask, face shield, and surgical cap). The surgical gown and first pair of gloves are discarded in the operating room.

5. After this, the surgical team leaves the operating theater and removes the face shield using the head band only without allowing the visor to touch the face, arms, or scrubs. The face mask is now discarded and full hand hygiene is undertaken.

\section{Neonatal Intensive Care Unit}

When a procedure is performed in NICU, there is a designated area within the NICU, large enough to admit a distance of
$2 \mathrm{~m}$ between the cots. Same principles of theater procedures are applied.

\section{Pediatric Patients and Individual Team Challenges}

\section{The Pediatric Patient}

The pediatric patient ranges from a newborn to the age of 16 years. The surgical procedures on the NICU will not have any change in anxiety levels in the neonates vis-à-vis interaction to the surgical team during the pandemic. But this may be different in other age groups during the preoperative phase in the theaters. ${ }^{28}$ Small children already have a high level of anxiety as they are unable to comprehend the pathology or the procedures in details; with the anxiety being even higher during the pandemic as they meet staff in PPE while entering theaters, thereby unable to see faces clearly and unable to understand individual staff roles. While being comforted during induction of anesthesia, what makes it even more difficult for small children is the inability to observe theater staff facial gestures behind the masks and face shields. Another dimension to be added is the lack of speech clarity from theater team members using FF3 or other fitted masks. Older children should also be given more information during preassessments to alleviate anxiety before they enter the operating theater. We had considered, as a group of anesthesiologists, the possibility of needing a lower threshold for premedication. However, we have found that with thorough assessment of anxiety levels of the child and parent, clear communication of what all theater staff will look like, and limiting the number of staff present during induction of anesthesia, we have not needed to premedicate this group of children with any greater frequency than before. During the present pandemic and beyond, special attention will need to be paid to these aspects of perioperative management. The pediatric patient interaction with theater staff has gone rather unnoticed and underreported during the present pandemic.

\section{Individual Team Challenges}

\section{(i) Electronic Communication Devices}

Ideally items such as mobile phones and on-call bleep are kept out of theaters that are considered to be a potentially contaminated area. If carried inside, they will be contained within a plastic cover. The anesthetic room is the clean area where mobile phones and bleeps can be kept for the clean runner to attend to messages and communicate them verbally to the operating surgeons.

\section{(ii) Interteam Communication and Identification}

Team communication has been an issue as it is always not clear to understand speech through a fitted FF3 (or other fitted) mask. To overcome this issue, it is important to limit the number of personnel in the theater and also to speak louder than usual so that the sentences can be comprehended. Personal identification of theater staff is another issue during the pandemic as name tags/ID cards are concealed with PPE. Name printed on self-sticking labels could 
either be attached to the top of the face shield at the time of donning or names can be written directly onto the gown using sterile marker pens.

\section{(iii) Optimizing Vision through Face Shields}

Another issue faced by the operating team is the extra glare caused by the theater lights falling on the face shields. This issue causes difficulties in visualization of the operative field especially in neonates and other procedures where the structures involved are of smaller dimensions. This issue has been partially addressed by head movement to dodge the light angles and obtain a better view of the operating field. Another issue with face shields during neonatal surgery is the close proximity of the operating team to the patient, which leads to frequent head clashing incidents among the operating team members.

\section{Conclusion}

This is a narrative review during the ongoing COVID-19 pandemic to streamline workflow of pediatric surgical patients in operating theaters and for theater teams involved in their management. Pediatric patient anxiety in theaters, aspects of communication, and optimizing vision during surgery during the pandemic have also been addressed.

\section{Conflict of Interest}

None declared.

\section{References}

1 National Health Commission of the People's Republic of China. Diagnosis and treatment project for pneumonia caused by novel coronavirus infection. Trial version 5. 2020; Accessed May 28, 2021 at: http://www.gov.cn/zhengce/zhengceku/2020-02/05/ 5474791/filesde44557832ad4be1929091dcbcfca891.pdf

2 Huang C, Wang Y, Li X, et al. Clinical features of patients infected with 2019 novel coronavirus in Wuhan, China. Lancet 2020;395 (10223):497-506

3 Chen N, Zhou M, Dong X, et al. Epidemiological and clinical characteristics of 99 cases of 2019 novel coronavirus pneumonia in Wuhan, China: a descriptive study. Lancet 2020;395(10223):507-513

4 Cheung KS, Hung IFN, Chan PPY, et al. Gastrointestinal manifestations of SARS-CoV-2 infection and virus load in fecal samples from the Hong Kong cohort and systematic review and metaanalysis. Gastroenterology 2020;159(01):81-95

5 FACS. org (Internet) COVID-19 Guidelines for Triage of Pediatric Patients. (Published 24 March 2020). Accessed May 28, 2021 at: https://www.facs.org/covid-19/clinical-guidance/elective-case/pediatric-surgery?fbclid=IwAR28d_VDVKDqGU7yUpW60003eg9nd5NJjeH6U5tFy0JjjXCAyf5icjirslo

6 Updated Intercollegiate General Surgery Guidance on COVID-19 30th May 2020. Accessed May 28, 2021 at: https://www.rcseng. ac.uk/coronavirus/joint-guidance-for-surgeons-v2/7

7 GOV.uk (Internet) Recommended PPE for healthcare workers by secondary care inpatient clinical setting, NHS and independent sector. (V1 Published 14 May 2020, accessed 23 May 2020). Accessed May 28, 2021 at: https://assets.publishing.service.gov.uk/government/uploads/system/uploads/attachment_data/file/ 886707/T1_poster_Recommended_PPE_for_healthcare_workers_by_secondary_care_clinical_context.pdf

8 Dong Y, Mo X, Hu Y, et al. Epidemiology of Covid-19 among children in China. Pediatrics 2020;145(06):e20200702
9 Hindson J. COVID-19: faecal-oral transmission? Nat Rev Gastroenterol Hepatol 2020;17(05):259

$10 \mathrm{Xu} \mathrm{Y,} \mathrm{Li} \mathrm{X,} \mathrm{Zhu} \mathrm{B,} \mathrm{et} \mathrm{al.} \mathrm{Characteristics} \mathrm{of} \mathrm{pediatric} \mathrm{SARS-CoV-2}$ infection and potential evidence for persistent fecal viral shedding. Nat Med 2020;26(04):502-505

11 Ti LK, Ang LS, Foong TW, Ng BSW. What we do when a COVID-19 patient needs an operation: operating room preparation and guidance. Can J Anaesth 2020;67(06):756-758

12 Walsh CM, Fishman DS, Lerner DGNASPGHAN Endoscopy and Procedures Committee. Pediatric endoscopy in the era of coronavirus disease 2019: a North American Society for Pediatric Gastroenterology, Hepatology, and Nutrition Position Paper. J Pediatr Gastroenterol Nutr 2020;70(06):741-750

13 Al-Benna S. Infection control in operating theatres. J Perioper Pract 2012;22(10):318-322

14 Public Health England. COVID-19: Guidance for infection prevention and control in healthcare settings. Version 1.1. 27 /03/20. COVID-19: infection prevention and control guidance. 2020 Accessed May 28, 2021 at: https://www.gov.uk/government/ publications/wuhan-novel-coronavirus-infection-preventionand-control

15 Cook TM, Harrop-Griffiths W. Aerosol clearance times to better communicate safety after aerosol-generating procedures. Anaesthesia 2020;75(08):1122-1123

16 Porter J, Blau E, Gharagozloo F, et al. Society of Robotic Surgery review: recommendations regarding the risk of COVID-19 transmission during minimally invasive surgery. BJU Int 2020;126(02): 225-234

17 SAGES and EAES Recommendations Regarding Surgical Response to COVID-19 Crisis. Available at: https://www.sages.org/recommendations-surgical-response-covid-19/?fbcli\%20d\%C2\%BC\% 20IwAR2M139pV3bwWp9XYB\%202cfWyYgrnx\%20bks0W\% 203GZ_\%20sZ0GrGXVucZQULX\%20qOf7jfk

18 Intercollegiate General Surgery Guidance on COVID-19 UPDATE | The Royal College of Surgeons of Edinburgh. Accessed May 28, 2021 at: https://www.rcsed.ac.uk/news-public-affairs/news/2020/ march/intercollegiate-general-surgery-guidance-on-covid-19update

19 Saxena AK. SARS-CoV-2 pandemic and pediatric endoscopic surgery. J Ped Endosc Surg. 2020;2:51-53

20 Kwak HD, Kim SH, Seo YS, Song KJ. Detecting hepatitis B virus in surgical smoke emitted during laparoscopic surgery. Occup Environ Med 2016;73(12):857-863

21 Zheng MH, Boni L, Fingerhut A. Minimally invasive surgery and the novel coronavirus outbreak: lessons learned in China and Italy. Ann Surg 2020;272(01):e5-e6

22 Saxena AK. Pediatric endoscopic surgery during the ongoing SARS-CoV-2 pandemic. J Ped Endosc Surg; 2021. Doi: 10.1007/ s42804-021-00099-5

23 Royal College of Surgeons. Intercollegiate General Surgery Guidance on COVID-19. Available at: https://www.rcsed.ac.uk/newspublic-affairs/news/2020/march/intercollegiate-general-surgeryguidance-on-covid-19-update

24 van Doremalen N, Bushmaker T, Morris DH, et al. Aerosol and surface stability of SARS-CoV-2 as compared with SARS-CoV-1. N Engl J Med 2020;382(16):1564-1567

25 Alqadi GO, Saxena AK. Smoke and particulate filters in endoscopic surgery reviewed during the SARS-CoV-2. J Ped Endosc Surg. 2020;2:61-67

26 da Costa KM, Saxena AK. Coronavirus disease 2019 pandemic and identifying insufflators with desufflation mode and surgical smoke evacuators for safe CO2 removal. Asian J Endosc Surg 2020. Doi: 10.1111 /ases. 12834

27 Abdelbaset AA, Saxena AK. Gasless laparoscopy revisited during the SARS-CoV-2. J Ped Endosc Surg 2020;2:61-67

28 Fronk E, Billick SB. Pre-operative anxiety in pediatric surgery patients: multiple case study analysis with literature review. Psychiatr Q 2020;91(04):1439-1451 